\title{
The Equivalence of Convergence Results of Modified Mann and Ishikawa Iterations with Errors without Bounded Range Assumption
}

\author{
Zhiqun Xue, ${ }^{1}$ Yaning Wang, ${ }^{1}$ and Haiyun Zhou ${ }^{2}$ \\ ${ }^{1}$ Department of Mathematics and Physics, Shijiazhuang Tiedao University, Shijiazhuang 050043, China \\ ${ }^{2}$ Department of Mathematics, Shijiazhuang Mechanical Engineering College, Shijiazhuang 050003, China \\ Correspondence should be addressed to Zhiqun Xue, xuezhiqun@126.com
}

Received 15 August 2012; Accepted 27 August 2012

Academic Editor: Yongfu Su

Copyright (C) 2012 Zhiqun Xue et al. This is an open access article distributed under the Creative Commons Attribution License, which permits unrestricted use, distribution, and reproduction in any medium, provided the original work is properly cited.

Let $E$ be an arbitrary uniformly smooth real Banach space, let $D$ be a nonempty closed convex subset of $E$, and let $T: D \rightarrow D$ be a uniformly generalized Lipschitz generalized asymptotically $\Phi$-strongly pseudocontractive mapping with $q \in F(T) \neq \emptyset$. Let $\left\{a_{n}\right\},\left\{b_{n}\right\},\left\{c_{n}\right\},\left\{d_{n}\right\}$ be four real sequences in [0,1] and satisfy the conditions: (i) $a_{n}+c_{n} \leq 1, b_{n}+d_{n} \leq 1$; (ii) $a_{n}, b_{n}, d_{n} \rightarrow 0$ as $n \rightarrow \infty$ and $c_{n}=o\left(a_{n}\right)$; (iii) $\sum_{n=0}^{\infty} a_{n}=\infty$. For some $x_{0}, z_{0} \in D$, let $\left\{u_{n}\right\},\left\{v_{n}\right\},\left\{w_{n}\right\}$ be any bounded sequences in $D$, and let $\left\{x_{n}\right\},\left\{z_{n}\right\}$ be the modified Ishikawa and Mann iterative sequences with errors, respectively. Then the convergence of $\left\{x_{n}\right\}$ is equivalent to that of $\left\{z_{n}\right\}$.

\section{Introduction and Preliminary}

Let $E$ be a real Banach space and let $E^{*}$ be its dual space. The normalized duality mapping $J: E \rightarrow 2^{E^{*}}$ is defined by

$$
J(x)=\left\{f \in E^{*}:\langle x, f\rangle=\|x\|^{2}=\|f\|^{2}\right\}, \quad \forall x \in E,
$$

where $\langle\cdot, \cdot\rangle$ denotes the generalized duality pairing. It is well known that

(i) if $E$ is a smooth Banach space, then the mapping $J$ is single-valued;

(ii) $J(\alpha x)=\alpha J(x)$ for all $x \in E$ and $\alpha \in \mathfrak{R}$;

(iii) if $E$ is a uniformly smooth Banach space, then the mapping $J$ is uniformly continuous on any bounded subset of $E$. Throughout this paper, we denote that 
$j$ is the single-valued normalized duality mapping, $D$ is a nonempty closed convex subset of $E, T: D \rightarrow D$ is a mapping, and $T^{0}$ is the unit mapping $I$.

In 1972, Goebel and Kirk [1] introduced the class of asymptotically nonexpansive mappings as follows.

Definition 1.1. A mapping $T$ is said to be asymptotically nonexpansive if for each $x, y \in D$

$$
\left\|T^{n} x-T^{n} y\right\| \leq k_{n}\|x-y\|, \quad \forall n \geq 0,
$$

where $\left\{k_{n}\right\} \subset[1,+\infty)$ with $\lim _{n \rightarrow \infty} k_{n}=1$.

Schu [2], in 1991, gave the definition of asymptotically pseudocontractive mappings and proved the correlation results.

Definition 1.2. The mapping $T$ is called asymptotically pseudocontractive with the sequence $\left\{k_{n}\right\} \subset[1,+\infty)$ if and only if $\lim _{n \rightarrow \infty} k_{n}=1$, and for all $n \in N$ and all $x, y \in D$, there exists $j(x-y) \in J(x-y)$ such that

$$
\left\langle T^{n} x-T^{n} y, j(x-y)\right\rangle \leq k_{n}\|x-y\|^{2}
$$

It is easy to find that every asymptotically nonexpansive mapping is asymptotically pseudocontractive. However, the converse is not true in general. See example of [3].

Recently, Colao [4] combined the proof ideas of the papers of Chang [5] and C. E. Chidume and C. O. Chidume [6] and then showed the equivalent theorem results of the convergence between Mann and Ishikawa iterations with errors for generalized strongly asymptotically $\phi$-pseudocontractive mapping with bounded range. In fact, he proved the following theorem.

Theorem 1.3. Let $X$ be a uniformly smooth Banach space, and let $T: X \rightarrow X$ be generalized strongly asymptotically $\phi$-pseudocontractive mapping with fixed point $x^{*}$ and bounded range. Let $\left\{x_{n}\right\}$ and $\left\{z_{n}\right\}$ be the sequences defined by (1.4) and (1.5), respectively,

$$
\begin{array}{cc}
y_{n}=\left(1-\beta_{n}-\delta_{n}\right) x_{n}+\beta_{n} T^{n} x_{n}+\delta_{n} v_{n}, & n \geq 0, \\
x_{n+1}=\left(1-\alpha_{n}-\gamma_{n}\right) x_{n}+\alpha_{n} T^{n} y_{n}+\gamma_{n} u_{n}, & n \geq 0, \\
z_{n+1}=\left(1-\alpha_{n}-\gamma_{n}\right) z_{n}+\alpha_{n} T^{n} z_{n}+\gamma_{n} w_{n}, & n \geq 0,
\end{array}
$$

where $\left\{\alpha_{n}\right\},\left\{\gamma_{n}\right\},\left\{\beta_{n}\right\},\left\{\delta_{n}\right\} \subset[0,1]$ satisfy

(H1) $\lim _{n \rightarrow \infty} \alpha_{n}=\lim _{n \rightarrow \infty} \beta_{n}=\lim _{n \rightarrow \infty} \delta_{n}=0$ and $\gamma_{n}=o\left(\alpha_{n}\right)$,

(H2) $\sum_{n=1}^{\infty} \alpha_{n}=\infty$,

and the sequences $\left\{u_{n}\right\},\left\{v_{n}\right\},\left\{w_{n}\right\}$ are bounded in $X$, then for any initial point $z_{0}, x_{0} \in X$, the following two assertions are equivalent.

(1) The modified Ishikawa iteration sequence with errors (1.4) converges to $x^{*}$;

(2) The modified Mann iteration sequence with errors (1.5) converges to $x^{*}$. 
The aim of this paper is to prove the equivalence of convergent results of above Ishikawa and Mann iterations with errors for generalized asymptotically $\Phi$-strongly pseudocontractive mappings without bounded range assumptions in uniformly smooth real Banach spaces. For this, we need the following concepts and lemmas.

Definition 1.4 (see [4]). The mapping $T$ is called generalized asymptotically $\Phi$-strongly pseudocontractive if

$$
\left\langle T^{n} x-T^{n} y, j(x-y)\right\rangle \leq k_{n}\|x-y\|^{2}-\Phi(\|x-y\|), \quad n \geq 0
$$

where $j(x-y) \in J(x-y),\left\{k_{n}\right\} \subset[1,+\infty)$ is converging to one and $\Phi:[0,+\infty) \rightarrow[0,+\infty)$ is strictly increasing continuous function with $\Phi(0)=0$.

Definition 1.5 (see [4]). For arbitrary given $x_{0} \in D$, modified Ishikawa iterative process with errors $\left\{x_{n}\right\}_{n=0}^{\infty}$ defined by

$$
\begin{aligned}
& y_{n}=\left(1-b_{n}-d_{n}\right) x_{n}+b_{n} T^{n} x_{n}+d_{n} w_{n}, \quad n \geq 0, \\
& x_{n+1}=\left(1-a_{n}-c_{n}\right) x_{n}+a_{n} T^{n} y_{n}+c_{n} v_{n}, \quad n \geq 0,
\end{aligned}
$$

where $\left\{v_{n}\right\},\left\{w_{n}\right\}$ are any bounded sequences in $D ;\left\{a_{n}\right\},\left\{b_{n}\right\},\left\{c_{n}\right\},\left\{d_{n}\right\}$ are four real sequences in $[0,1]$ and satisfy $a_{n}+c_{n} \leq 1, b_{n}+d_{n} \leq 1$, for all $n \geq 0$. If $b_{n}=d_{n}=0$, we define modified Mann iterative process with errors $\left\{z_{n}\right\}$ by

$$
z_{n+1}=\left(1-a_{n}-c_{n}\right) z_{n}+a_{n} T^{n} z_{n}+c_{n} u_{n}, \quad n \geq 0,
$$

where $\left\{u_{n}\right\}$ is any bounded sequence in $D$.

Lemma 1.6 (see [7]). Let E be a uniformly smooth real Banach space and let $J: E \rightarrow 2^{E^{*}}$ be a normalized duality mapping. Then

$$
\|x+y\|^{2} \leq\|x\|^{2}+2\langle y, J(x+y)\rangle
$$

for all $x, y \in E$.

Lemma 1.7 (see [8]). Let $\left\{\rho_{n}\right\}_{n=0}^{\infty}$ be a nonnegative sequence which satisfies the following inequality:

$$
\rho_{n+1} \leq\left(1-\lambda_{n}\right) \rho_{n}+\sigma_{n}, \quad n \geq 0
$$

where $\lambda_{n} \in[0,1]$ with $\sum_{n=0}^{\infty} \lambda_{n}=\infty, \sigma_{n}=o\left(\lambda_{n}\right)$. Then $\rho_{n} \rightarrow 0$ as $n \rightarrow \infty$.

\section{Main Results}

First of all, we give a new concept. 
Definition 2.1. A mapping $T: D \rightarrow D$ is called uniformly generalized Lipschitz if there exists a constant $L>0$ such that

$$
\left\|T^{n} x-T^{n} y\right\| \leq L(1+\|x-y\|), \quad \forall x, y \in D, \forall n \geq 0 .
$$

It is mentioned to notice that if $T$ has bounded range, then it is uniformly generalized Lipschitz. In fact, since $R\left(T^{n}\right) \subseteq R(T)$, then $\sup _{x \in D}\left\{\left\|T^{n} x\right\|\right\} \leq \sup _{x \in D}\{\|T x\|\}=M_{1}$, thus $\left\|T^{n} x-T^{n} y\right\| \leq 2 M_{1} \leq L(1+\|x-y\|)$, where $L=2 M_{1}$. On the contrary, it is not true in general (See [6]).

In the following, we prove the main theorems of this paper.

Theorem 2.2. Let $E$ be an arbitrary uniformly smooth real Banach space, let $D$ be a nonempty closed convex subset of $E$, and let $T: D \rightarrow D$ be a uniformly generalized Lipschitz generalized asymptotically $\Phi$-strongly pseudocontractive mapping with $q \in F(T) \neq \emptyset$. Let $\left\{a_{n}\right\},\left\{b_{n}\right\},\left\{c_{n}\right\},\left\{d_{n}\right\}$ be four real sequences in $[0,1]$ and satisfy the following conditions:

(i) $a_{n}+c_{n} \leq 1, b_{n}+d_{n} \leq 1$;

(ii) $a_{n}, b_{n}, d_{n} \rightarrow 0$ as $n \rightarrow \infty$ and $c_{n}=o\left(a_{n}\right)$;

(iii) $\sum_{n=0}^{\infty} a_{n}=\infty$.

For some $x_{0}, z_{0} \in D$, let $\left\{u_{n}\right\},\left\{v_{n}\right\},\left\{w_{n}\right\}$ be any bounded sequences in $D$, and let $\left\{x_{n}\right\}$ and $\left\{z_{n}\right\}$ be Ishikawa and Mann iterative sequences with errors defined by (1.7) and (1.8), respectively. Then the following conclusions are equivalent:

(1) $\left\{x_{n}\right\}$ converges strongly to the unique fixed point $q$ of $T$;

(2) $\left\{z_{n}\right\}$ converges strongly to the unique fixed point $q$ of $T$.

Proof. (1) $\Rightarrow(2)$ is obvious, that is, let $b_{n}=d_{n}=0$, (1.7) turns into (1.8). We only need to show that $(2) \Rightarrow(1)$. Since $T: D \rightarrow D$ is a uniformly generalized Lipschitz generalized asymptotically $\Phi$-strongly pseudocontractive mapping, then there exists a strictly increasing continuous function $\Phi:[0,+\infty) \rightarrow[0,+\infty)$ with $\Phi(0)=0$ such that

$$
\left\langle T^{n} x-T^{n} y, J(x-y)\right\rangle \leq k_{n}\|x-y\|^{2}-\Phi(\|x-y\|),
$$

that is,

$$
\begin{gathered}
\left\langle\left(k_{n} I-T^{n}\right) x-\left(k_{n} I-T^{n}\right) y, J(x-y)\right\rangle \geq \Phi(\|x-y\|), \\
\left\|T^{n} x-T^{n} y\right\| \leq L(1+\|x-y\|),
\end{gathered}
$$

for any $x, y \in D$. For convenience, denote $k=\sup _{n}\left\{k_{n}\right\}$.

Step 1 . There exists $x_{0} \in D$ and $x_{0} \neq T x_{0}$ such that $r_{0}=(k+L)\left\|x_{0}-q\right\|^{2}+L\left\|x_{0}-q\right\| \in R(\Phi)$ (range of $\Phi)$.

Indeed, if $\Phi(r) \rightarrow+\infty$ as $r \rightarrow+\infty$, then $r_{0} \in R(\Phi)$; if $\sup \{\Phi(r): r \in[0,+\infty)\}=r_{1}<$ $+\infty$ with $r_{1}<r_{0}$, then, for $q \in D$, there exists a sequence $\left\{v_{n}\right\}$ in $D$ such that $v_{n} \rightarrow q$ as $n \rightarrow \infty$ with $v_{n} \neq q$. Furthermore, there exists a natural number $n_{0}$ such that $(k+L)\left\|v_{n}-q\right\|^{2}+L\left\|v_{n}-q\right\|<$ 
$r_{1} / 2$ for $n \geq n_{0}$, then we redefine $x_{0}, r_{0}$ such that $x_{0}=v_{n_{0}}, r_{0}=(k+L)\left\|x_{0}-q\right\|^{2}+L\left\|x_{0}-q\right\| \in R(\Phi)$. Hence, it is to ensure that $\Phi^{-1}\left(r_{0}\right)$ is well defined.

Step 2. For any $n \geq 0,\left\{x_{n}\right\}$ is a bounded sequence.

Set $R=\Phi^{-1}\left(r_{0}\right)$. From (2.3), we have

$$
\left\langle k_{n}\left(x_{0}-q\right)-\left(T^{n} x_{0}-q\right), J\left(x_{0}-q\right)\right\rangle \geq \Phi\left(\left\|x_{0}-q\right\|\right),
$$

that is, $(k+L)\left\|x_{0}-q\right\|^{2}+L\left\|x_{0}-q\right\| \geq \Phi\left(\left\|x_{0}-q\right\|\right)$. Thus, we obtain that $\left\|x_{0}-q\right\| \leq R$. Denote

$$
\begin{gathered}
B_{1}=\{x \in D:\|x-q\| \leq R\}, \\
B_{2}=\{x \in D:\|x-q\| \leq 2 R\}, \\
M=\sup _{n}\left\{\left\|v_{n}-q\right\|\right\}+\sup _{n}\left\{\left\|w_{n}-q\right\|\right\} .
\end{gathered}
$$

Next, we want to prove that $x_{n} \in B_{1}$ for any $n \geq 0$ by induction. If $n=0$, then $x_{0} \in B_{1}$. Now we assume that it holds for some $n$, that is, $x_{n} \in B_{1}$. We prove that $x_{n+1} \in B_{1}$. Suppose that it is not the case, then $\left\|x_{n+1}-q\right\|>R$. Since $J$ is uniformly continuous on bounded subset of $E$, then, for $\epsilon_{0}=\Phi(R / 4) / 24 L(1+2 R)$, there exists $\delta>0$ such that $\|J x-J y\|<\epsilon_{0}$ when $\|x-y\|<\delta$, for all $x, y \in B_{2}$. Now denote

$$
\begin{aligned}
\tau_{0}=\min \left\{\frac{R}{2[L(1+2 R)+2 R+M]}, \frac{R}{4[L(1+R)+2 R+M]},\right. \\
\left.\frac{\delta}{2[L(1+2 R)+2 R+M]}, \frac{\Phi(R / 4)}{24 R^{2}}, \frac{\Phi(R / 4)}{24 L(1+2 R)}, \frac{\Phi(R / 4)}{48 M R}\right\} .
\end{aligned}
$$

Since $a_{n}, b_{n}, c_{n}, d_{n} \rightarrow 0$ as $n \rightarrow \infty$, and $c_{n}=o\left(a_{n}\right)$, without loss of generality, we assume that $0 \leq a_{n}, b_{n}, c_{n}, d_{n} \leq \tau_{0}, c_{n}<a_{n} \tau_{0}$ for any $n \geq 0$. Then we obtain the following estimates:

$$
\begin{aligned}
\left\|T^{n} x_{n}-q\right\| & \leq L\left(1+\left\|x_{n}-q\right\|\right) \\
& \leq L(1+R), \\
\left\|y_{n}-q\right\| & \leq\left(1-b_{n}-d_{n}\right)\left\|x_{n}-q\right\|+b_{n}\left\|T^{n} x_{n}-q\right\|+d_{n}\left\|w_{n}-q\right\| \\
& \leq R+b_{n} L\left(1+\left\|x_{n}-q\right\|\right)+d_{n} M \\
& \leq R+b_{n} L(1+R)+d_{n} M \\
& \leq R+\tau_{0}[L(1+R)+M] \\
& \leq 2 R, \\
\left\|T^{n} y_{n}-q\right\| & \leq L\left(1+\left\|y_{n}-q\right\|\right) \\
& \leq L(1+2 R),
\end{aligned}
$$




$$
\begin{aligned}
& \left\|x_{n}-T^{n} x_{n}\right\| \leq\left\|x_{n}-q\right\|+\left\|T^{n} x_{n}-q\right\| \\
& \leq L+(1+L)\left\|x_{n}-q\right\| \\
& \leq L+(1+L) R, \\
& \left\|\left(x_{n}-q\right)-\left(y_{n}-q\right)\right\| \leq b_{n}\left\|x_{n}-T^{n} x_{n}\right\|+d_{n}\left[\left\|w_{n}-q\right\|+\left\|x_{n}-q\right\|\right] \\
& \leq b_{n}[L+(1+L) R]+d_{n}(M+R) \\
& \leq \tau_{0}[L(1+R)+2 R+M] \\
& \leq \tau_{0}[L(1+2 R)+2 R+M] \\
& \leq \frac{\delta}{2}<\delta, \\
& \left\|x_{n}-q\right\| \geq\left\|x_{n+1}-q\right\|-a_{n}\left\|T^{n} y_{n}-x_{n}\right\|-c_{n}\left\|v_{n}-x_{n}\right\| \\
& \geq\left\|x_{n+1}-q\right\|-a_{n}\left[\left\|T^{n} y_{n}-q\right\|+\left\|x_{n}-q\right\|\right]-c_{n}\left[\left\|x_{n}-q\right\|+\left\|v_{n}-q\right\|\right] \\
& >R-a_{n}[L(1+2 R)+R]-c_{n}(R+M) \\
& \geq R-\tau_{0}[L(1+2 R)+M+2 R] \\
& \geq R-\frac{R}{2}=\frac{R}{2}, \\
& \left\|y_{n}-q\right\| \geq\left\|x_{n}-q\right\|-b_{n}\left\|T^{n} x_{n}-x_{n}\right\|-d_{n}\left\|x_{n}-w_{n}\right\| \\
& \geq\left\|x_{n}-q\right\|-b_{n}[L+(1+L) R]-d_{n}\left[\left\|x_{n}-q\right\|+\left\|w_{n}-q\right\|\right] \\
& \geq\left\|x_{n}-q\right\|-b_{n}[L+(1+L) R]-d_{n}(R+M) \\
& \geq\left\|x_{n}-q\right\|-\tau_{0}[L(1+R)+2 R+M] \\
& >\frac{R}{2}-\frac{R}{4}=\frac{R}{4}, \\
& \left\|x_{n+1}-q\right\| \leq\left(1-a_{n}-c_{n}\right)\left\|x_{n}-q\right\|+a_{n}\left\|T^{n} y_{n}-q\right\|+c_{n}\left\|v_{n}-q\right\| \\
& \leq R+\tau_{0}[L(1+2 R)+M] \\
& \leq 2 R \text {, } \\
& \left\|\left(x_{n+1}-q\right)-\left(x_{n}-q\right)\right\| \leq a_{n}\left\|T^{n} y_{n}-x_{n}\right\|+c_{n}\left\|u_{n}-x_{n}\right\| \\
& \leq a_{n}\left[\left\|T^{n} y_{n}-q\right\|+\left\|x_{n}-q\right\|\right]+c_{n}\left[\left\|v_{n}-q\right\|+\left\|x_{n}-q\right\|\right] \\
& \leq a_{n}[L(1+2 R)+R]+c_{n}(M+R) \\
& \leq \tau_{0}[L(1+2 R)+2 R+M] \\
& \leq \frac{\delta}{2}<\delta \text {. }
\end{aligned}
$$

Hence, $\left\|J\left(x_{n}-q\right)-J\left(y_{n}-q\right)\right\|<\epsilon_{0} ;\left\|J\left(x_{n+1}-q\right)-J\left(x_{n}-q\right)\right\|<\epsilon_{0}$. 
Using Lemma 1.6 and formulas above, we obtain

$$
\begin{aligned}
\left\|x_{n+1}-q\right\|^{2} \leq & \left(1-a_{n}-c_{n}\right)^{2}\left\|x_{n}-q\right\|^{2}+2 a_{n}\left\langle T^{n} y_{n}-q, J\left(x_{n+1}-q\right)\right\rangle \\
& +2 c_{n}\left\langle u_{n}-q, J\left(x_{n+1}-q\right)\right\rangle \\
\leq & \left(1-a_{n}\right)^{2}\left\|x_{n}-q\right\|^{2}+2 a_{n}\left\langle T^{n} y_{n}-q, J\left(x_{n+1}-q\right)-J\left(x_{n}-q\right)\right\rangle \\
& +2 a_{n}\left\langle T^{n} y_{n}-q, J\left(x_{n}-q\right)-J\left(y_{n}-q\right)\right\rangle \\
& +2 a_{n}\left\langle T^{n} y_{n}-q, J\left(y_{n}-q\right)\right\rangle+2 c_{n}\left\langle u_{n}-q, J\left(x_{n+1}-q\right)\right\rangle \\
\leq & \left(1-a_{n}\right)^{2}\left\|x_{n}-q\right\|^{2}+2 a_{n}\left\|T^{n} y_{n}-q\right\| \cdot\left\|J\left(x_{n+1}-q\right)-J\left(x_{n}-q\right)\right\| \\
& +2 a_{n}\left\|T^{n} y_{n}-q\right\| \cdot\left\|J\left(x_{n}-q\right)-J\left(y_{n}-q\right)\right\| \\
& +2 a_{n}\left[\left\|y_{n}-q\right\|^{2}-\Phi\left(\left\|y_{n}-q\right\|\right)\right]+2 c_{n}\left\|u_{n}-q\right\| \cdot\left\|x_{n+1}-q\right\| \\
\leq & \left(1-a_{n}\right)^{2} R^{2}+4 a_{n} L(1+2 R) \epsilon_{0}+2 a_{n}\left[\left\|y_{n}-q\right\|^{2}-\Phi\left(\left\|y_{n}-q\right\|\right)\right] \\
& +4 c_{n} M R, \\
\| & \left(1-b_{n}-d_{n}\right)^{2}\left\|x_{n}-q\right\|^{2}+2 b_{n}\left\langle T^{n} x_{n}-q, J\left(y_{n}-q\right)\right\rangle \\
& +2 d_{n}\left\langle w_{n}-q, J\left(y_{n}-q\right)\right\rangle \\
\leq & \left\|x_{n}-q\right\|^{2}+2 b_{n}\left\langle T^{n} x_{n}-q, J\left(y_{n}-q\right)-J\left(x_{n}-q\right)\right\rangle \\
& +2 b_{n}\left\langle T^{n} x_{n}-q, J\left(x_{n}-q\right)\right\rangle+2 d_{n}\left\|w_{n}-q\right\| \cdot\left\|y_{n}-q\right\| \\
\leq & \left\|x_{n}-q\right\|^{2}+2 b_{n}\left\|T_{n}^{x}-q\right\| \cdot\left\|J\left(y_{n}-q\right)-J\left(x_{n}-q\right)\right\| \\
& +2 b_{n}\left[\left\|x_{n}-q\right\|^{2}-\Phi\left(\left\|x_{n}-q\right\|\right)\right]+2 d_{n}\left\|w_{n}-q\right\| \cdot\left\|y_{n}-q\right\| \\
\leq & R^{2}+2 b_{n} L(1+R) \epsilon_{0}+2 b_{n} R^{2}+4 d_{n} M R . \\
2 &
\end{aligned}
$$

Substitute (2.10) into (2.9)

$$
\begin{aligned}
\left\|x_{n+1}-q\right\|^{2} \leq & \left(1-a_{n}\right)^{2} R^{2}+4 a_{n} L(1+2 R) \epsilon_{0}+2 a_{n}\left[R^{2}+2 b_{n} L(1+R) \epsilon_{0}+2 b_{n} R^{2}+4 d_{n} M R\right] \\
& -2 a_{n} \Phi\left(\left\|y_{n}-q\right\|\right)+4 c_{n} M R \\
\leq & R^{2}+a_{n}^{2} R^{2}+4 a_{n} L(1+2 R) \epsilon_{0}+2 a_{n}\left[2 b_{n} L(1+R) \epsilon_{0}+2 b_{n} R^{2}+4 d_{n} M R\right] \\
& -2 a_{n} \Phi\left(\frac{R}{4}\right)+4 c_{n} M R
\end{aligned}
$$




$$
\begin{aligned}
= & R^{2}+2 a_{n}\left[\frac{a_{n}}{2} R^{2}+2 L(1+2 R) \epsilon_{0}+2 b_{n} L(1+R) \epsilon_{0}+2 b_{n} R^{2}+4 d_{n} M R+\frac{2 c_{n} M R}{a_{n}}\right] \\
& -2 a_{n} \Phi\left(\frac{R}{4}\right) \\
\leq & R^{2}+2 a_{n}\left[\frac{\Phi(R / 4)}{2}-\Phi\left(\frac{R}{4}\right)\right] \\
\leq & R^{2}-\Phi\left(\frac{R}{4}\right) a_{n} \\
\leq & R^{2}
\end{aligned}
$$

this is a contradiction. Thus $x_{n+1} \in B_{1}$, that is, $\left\{x_{n}\right\}$ is a bounded sequence. So $\left\{y_{n}\right\}$, $\left\{T^{n} y_{n}\right\},\left\{T^{n} x_{n}\right\}$ are all bounded sequences. Since $\left\|z_{n}-q\right\| \rightarrow 0$ as $n \rightarrow \infty$, without loss of generality, we let $\left\|z_{n}-q\right\| \leq 1$. Therefore, $\left\|x_{n}-z_{n}\right\|$ is also bounded.

Step 3. We want to prove $\left\|x_{n}-z_{n}\right\| \rightarrow 0$ as $n \rightarrow \infty$.

Set $M_{0}=\max \left\{\sup _{n}\left\|T^{n} y_{n}-T^{n} z_{n}\right\|, \sup _{n}\left\|v_{n}-u_{n}\right\|, \sup _{n}\left\|x_{n}-z_{n}\right\|, \sup _{n} \| T^{n} x_{n}-\right.$ $\left.x_{n}\left\|, \sup _{n}\right\| w_{n}-x_{n}\left\|, \sup _{n}\right\| y_{n}-z_{n}\left\|, \sup _{n}\right\| v_{n}-x_{n} \|\right\}$.

Again using Lemma 1.6, we have

$$
\begin{aligned}
\left\|x_{n+1}-z_{n+1}\right\|^{2} \leq & \left(1-a_{n}-c_{n}\right)^{2}\left\|x_{n}-z_{n}\right\|^{2}+2 a_{n}\left\langle T^{n} y_{n}-T^{n} z_{n} J\left(x_{n+1}-z_{n+1}\right)\right\rangle \\
& +2 c_{n}\left\langle v_{n}-u_{n} J\left(x_{n+1}-z_{n+1}\right)\right\rangle \\
\leq & \left(1-a_{n}\right)^{2}\left\|x_{n}-z_{n}\right\|^{2}+2 a_{n}\left\langle T^{n} y_{n}-T^{n} z_{n}, J\left(x_{n+1}-z_{n+1}\right)-J\left(x_{n}-z_{n}\right)\right\rangle \\
& +2 a_{n}\left\langle T^{n} y_{n}-T^{n} z_{n}, J\left(x_{n}-z_{n}\right)-J\left(y_{n}-z_{n}\right)\right\rangle \\
& +2 a_{n}\left\langle T^{n} y_{n}-T^{n} z_{n}, J\left(y_{n}-z_{n}\right)\right\rangle+2 c_{n}\left\|v_{n}-u_{n}\right\| \cdot\left\|x_{n+1}-z_{n+1}\right\| \\
\leq & \left(1-a_{n}\right)^{2}\left\|x_{n}-z_{n}\right\|^{2}+2 a_{n} M_{0} A_{n}+2 a_{n} M_{0} B_{n} \\
& +2 a_{n}\left[\left\|y_{n}-z_{n}\right\|^{2}-\Phi\left(\left\|y_{n}-z_{n}\right\|\right)\right]+2 c_{n} M_{0}^{2}, \\
\left\|y_{n}-z_{n}\right\|^{2} \leq & \left\|x_{n}-z_{n}\right\|^{2}+2 b_{n}\left\langle T^{n} x_{n}-x_{n}, J\left(y_{n}-z_{n}\right)\right\rangle \\
& +2 d_{n}\left\langle w_{n}-x_{n} J\left(y_{n}-z_{n}\right)\right\rangle \\
\leq & \left\|x_{n}-z_{n}\right\|^{2}+2 b_{n} M_{0}^{2}+2 d_{n} M_{0}^{2},
\end{aligned}
$$

where $A_{n}=\left\|J\left(x_{n+1}-z_{n+1}\right)-J\left(x_{n}-z_{n}\right)\right\|, B_{n}=\left\|J\left(x_{n}-z_{n}\right)-J\left(y_{n}-z_{n}\right)\right\|$, and $A_{n}, B_{n} \rightarrow 0$ as $n \rightarrow \infty$. 
Taking place (2.13) into (2.12), we have

$$
\begin{aligned}
\left\|x_{n+1}-z_{n+1}\right\|^{2} \leq & \left(1-a_{n}\right)^{2}\left\|x_{n}-z_{n}\right\|^{2}+2 a_{n} M_{0} A_{n}+2 a_{n} M_{0} B_{n} \\
& +2 a_{n}\left[\left\|x_{n}-z_{n}\right\|^{2}+2 b_{n} M_{0}^{2}+2 d_{n} M_{0}^{2}-\Phi\left(\left\|y_{n}-z_{n}\right\|\right)\right]+2 c_{n} M_{0}^{2} \\
\leq & \left\|x_{n}-z_{n}\right\|^{2}+a_{n}^{2} M_{0}^{2}+2 a_{n} M_{0} A_{n}+2 a_{n} M_{0} B_{n}+4 a_{n} b_{n} M_{0}^{2}+4 a_{n} d_{n} M_{0}^{2} \\
& -2 a_{n} \Phi\left(\left\|y_{n}-z_{n}\right\|\right)+2 c_{n} M_{0}^{2} \\
= & \left\|x_{n}-z_{n}\right\|^{2}+2 a_{n}\left[C_{n}-2 a_{n} \Phi\left(\left\|y_{n}-z_{n}\right\|\right)\right]
\end{aligned}
$$

where $C_{n}=a_{n} M_{0}^{2} / 2+M_{0} A_{n}+M_{0} B_{n}+2 b_{n} M_{0}^{2}+2 d_{n} M_{0}^{2}+c_{n} M_{0}^{2} / a_{n} \rightarrow 0$ as $n \rightarrow \infty$.

Set $\inf _{n \geq 0} \Phi\left(\left\|y_{n}-z_{n}\right\|\right) /\left(1+\left\|x_{n+1}-z_{n+1}\right\|^{2}\right)=\lambda$, then $\lambda=0$. If it is not the case, we assume that $\lambda>0$. Let $0<\gamma<\min \{1, \lambda\}$, then $\Phi\left(\left\|y_{n}-z_{n}\right\|\right) /\left(1+\left\|x_{n+1}-z_{n+1}\right\|^{2}\right) \geq \gamma$, that is, $\Phi\left(\left\|y_{n}-z_{n}\right\|\right) \geq \gamma+\gamma\left\|x_{n+1}-z_{n+1}\right\|^{2} \geq \gamma\left\|x_{n+1}-z_{n+1}\right\|^{2}$. Thus, from (2.14) that

$$
\left\|x_{n+1}-z_{n+1}\right\|^{2} \leq\left\|x_{n}-z_{n}\right\|^{2}+2 a_{n}\left(C_{n}-\gamma\left\|x_{n+1}-z_{n+1}\right\|^{2}\right),
$$

which implies that

$$
\begin{aligned}
\left\|x_{n+1}-z_{n+1}\right\|^{2} & \leq \frac{1}{1+2 a_{n} \gamma}\left\|x_{n}-z_{n}\right\|^{2}+\frac{2 a_{n} C_{n}}{1+2 a_{n} \gamma} \\
& =\left(1-\frac{2 a_{n} \gamma}{1+2 a_{n} \gamma}\right)\left\|x_{n}-z_{n}\right\|^{2}+\frac{2 a_{n} C_{n}}{1+2 a_{n} \gamma} .
\end{aligned}
$$

Let $\rho_{n}=\left\|x_{n}-z_{n}\right\|^{2}, \lambda_{n}=2 a_{n} \gamma /\left(1+2 a_{n} \gamma\right), \sigma_{n}=2 a_{n} C_{n} /\left(1+2 a_{n} \gamma\right)$. Then we get that

$$
\rho_{n+1} \leq\left(1-\lambda_{n}\right) \rho_{n}+\sigma_{n}
$$

Applying Lemma 1.7, we get that $\rho_{n} \rightarrow 0$ as $n \rightarrow \infty$. This is a contradiction and so $\lambda=0$. Therefore, there exists an infinite subsequence such that $\Phi\left(\left\|y_{n_{i}}-z_{n_{i}}\right\|\right) /\left(1+\left\|x_{n_{i}+1}-z_{n_{i}+1}\right\|^{2}\right) \rightarrow$ 0 as $i \rightarrow \infty$. Since $0 \leq \Phi\left(\left\|y_{n_{i}}-z_{n_{i}}\right\|\right) /\left(1+M_{0}^{2}\right) \leq \Phi\left(\left\|y_{n_{i}}-z_{n_{i}}\right\|\right) /\left(1+\left\|x_{n_{i}+1}-z_{n_{i}+1}\right\|^{2}\right)$, then $\Phi\left(\left\|y_{n_{i}}-z_{n_{i}}\right\|\right) \rightarrow 0$ as $i \rightarrow \infty$. In view of the strictly increasing and continuity of $\Phi$, we have $\left\|y_{n_{i}}-z_{n_{i}}\right\| \rightarrow 0$ as $i \rightarrow \infty$. From (1.7), we have

$$
\left\|x_{n_{i}}-z_{n_{i}}\right\| \leq\left\|y_{n_{i}}-z_{n_{i}}\right\|+b_{n_{i}}\left\|x_{n_{i}}-T x_{n_{i}}\right\|+c_{n_{i}}\left\|x_{n_{i}}-w_{n_{i}}\right\| \longrightarrow 0
$$

as $i \rightarrow \infty$. Next we want to prove $\left\|x_{n}-z_{n}\right\| \rightarrow 0$ as $n \rightarrow \infty$. Let for all $\varepsilon \in(0,1)$, there exists $n_{i_{0}}$ such that $\left\|x_{n_{i}}-z_{n_{i}}\right\|<\epsilon, a_{n}, a_{n_{i}}<\min \left\{\epsilon / 4 L\left(1+M_{0}\right), \epsilon / 8 M_{0}\right\}, c_{n}, c_{n_{i}}<\epsilon / 16 M_{0}, b_{n}, d_{n}, b_{n_{i}}, d_{n_{i}}<$ $\epsilon / 8 M_{0}, C_{n}, C_{n_{i}}<\Phi(\epsilon / 4) / 2$, for any $n_{i}, n \geq n_{i_{0}}$. First, we want to prove $\left\|x_{n_{i}+1}-z_{n_{i}+1}\right\|<\epsilon$. 
Suppose it is not this case, then $\left\|x_{n_{i}+1}-z_{n_{i}+1}\right\| \geq \epsilon$. Using (1.7), we may get the following estimates:

$$
\begin{aligned}
\left\|x_{n_{i}}-z_{n_{i}}\right\| \geq & \left\|x_{n_{i}+1}-z_{n_{i}+1}\right\|-a_{n_{i}}\left\|T^{n} y_{n_{i}}-T^{n} z_{n_{i}}\right\|-a_{n_{i}}\left\|x_{n_{i}}-z_{n_{i}}\right\| \\
& -c_{n_{i}}\left\|v_{n_{i}}-u_{n_{i}}\right\|-c_{n_{i}}\left\|x_{n_{i}}-z_{n_{i}}\right\| \\
\geq & \epsilon-a_{n_{i}} L\left(1+M_{0}\right)-\left(a_{n_{i}}+2 c_{n_{i}}\right) M_{0} \\
> & \frac{\epsilon}{2} \\
\left\|y_{n_{i}}-z_{n_{i}}\right\| \geq & \left\|x_{n_{i}}-z_{n_{i}}\right\|-b_{n_{i}}\left\|T^{n} x_{n_{i}}-x_{n_{i}}\right\|-d_{n_{i}}\left\|v_{n_{i}}-x_{n_{i}}\right\| \\
\geq & \frac{\epsilon}{2}-\left(b_{n_{i}}+d_{n_{i}}\right) M_{0} \\
> & \frac{\epsilon}{4} .
\end{aligned}
$$

Since $\Phi$ is strictly increasing, then (2.20) leads to $\Phi\left(\left\|y_{n_{i}}-z_{n_{i}}\right\|\right) \geq \Phi(\epsilon / 4)$. From (2.14), we have

$$
\begin{aligned}
\left\|x_{n_{i}+1}-z_{n_{i}+1}\right\|^{2} & \leq\left\|x_{n_{i}}-z_{n_{i}}\right\|^{2}+2 a_{n_{i}}\left[C_{n_{i}}-\Phi\left(\left\|y_{n_{i}}-z_{n_{i}}\right\|\right)\right] \\
& <\epsilon^{2}+2 a_{n_{i}}\left[\frac{1}{2} \Phi\left(\frac{\epsilon}{4}\right)-\Phi\left(\frac{\epsilon}{4}\right)\right] \\
& \leq \epsilon^{2}-\Phi\left(\frac{\epsilon}{4}\right) a_{n_{i}} \\
& \leq \epsilon^{2}
\end{aligned}
$$

is a contradiction. Hence, $\left\|x_{n_{i}+1}-z_{n_{i}+1}\right\|<\epsilon$. Suppose that $\left\|x_{n_{i}+m}-z_{n_{i}+m}\right\|<\epsilon$ holds. Repeating the above course, we can easily prove that $\left\|x_{n_{i}+m+1}-z_{n_{i}+m+1}\right\|<\epsilon$ holds. Therefore, for any $m$ and $n_{i} \geq n_{0}$, we obtain that $\left\|x_{n_{i}+m}-z_{n_{i}+m}\right\|<\epsilon$, which means $\left\|x_{n}-z_{n}\right\| \rightarrow 0$ as $n \rightarrow \infty$. This completes the proof. theorem.

In order to make the existence of Theorem 2.2 more meaningful, we give the following

Theorem 2.3. Let $E$ be an arbitrary uniformly smooth real Banach space, let $D$ be a nonempty closed convex subset of $E$, and let $T: D \rightarrow D$ be a uniformly generalized Lipschitz generalized asymptotically $\Phi$-strongly pseudocontractive mapping with $q \in F(T) \neq \emptyset$. Let $\left\{a_{n}\right\},\left\{c_{n}\right\}$ be two real sequences in $[0,1]$ and satisfy the conditions (i) $a_{n}+c_{n} \leq 1$; (ii) $a_{n} \rightarrow 0$ as $n \rightarrow \infty$ and $c_{n}=o\left(a_{n}\right)$; (iii) $\sum_{n=0}^{\infty} a_{n}=\infty$. For some $z_{0} \in D$, let $\left\{u_{n}\right\}$ be any bounded sequence in $D$ and let $\left\{z_{n}\right\}$ be modified Mann iterative sequence with errors defined by (1.8). Then $\left\{z_{n}\right\}$ converges strongly to the unique fixed point $q$ of $T$. 
Proof. Since $T: D \rightarrow D$ is a uniformly generalized Lipschitz generalized asymptotically $\Phi$-strongly pseudocontractive mapping, then there exists a strictly increasing continuous function $\Phi:[0,+\infty) \rightarrow[0,+\infty)$ with $\Phi(0)=0$ such that

$$
\begin{gathered}
\left\langle\left(k_{n} I-T^{n}\right) x-\left(k_{n} I-T^{n}\right) y, J(x-y)\right\rangle \geq \Phi(\|x-y\|), \\
\left\|T^{n} x-T^{n} y\right\| \leq L(1+\|x-y\|),
\end{gathered}
$$

for any $x, y \in D$.

Step 1 . There exists $z_{0} \in D$ and $z_{0} \neq T z_{0}$ such that $r_{0}=(k+L)\left\|z_{0}-q\right\|^{2}+L\left\|z_{0}-q\right\| \in R(\Phi)$, where $k=\sup _{n}\left\{k_{n}\right\}$. In fact, if $\Phi(r) \rightarrow+\infty$ as $r \rightarrow+\infty$, then $r_{0} \in R(\Phi)$; if $\sup \{\Phi(r)$ : $r \in[0,+\infty)\}=r_{1}<+\infty$ with $r_{1}<r_{0}$, then, for $q \in D$, there exists a sequence $\left\{v_{n}\right\}$ in $D$ such that $v_{n} \rightarrow q$ as $n \rightarrow \infty$ with $v_{n} \neq q$. Furthermore, there exists a natural number $n_{0}$ such that $(k+L)\left\|v_{n}-q\right\|^{2}+L\left\|v_{n}-q\right\|<\left(r_{1} / 2\right)$ for $n \geq n_{0}$, then we redefine $z_{0}, r_{0}$ such that $z_{0}=v_{n_{0}}, r_{0}=(k+L)\left\|z_{0}-q\right\|^{2}+L\left\|z_{0}-q\right\| \in R(\Phi)$.

Step 2. For any $n \geq 0,\left\{z_{n}\right\}$ is bounded.

Set $r=\Phi^{-1}\left(r_{0}\right)$, we have $\left\|x_{0}-q\right\| \leq R$. Let $B_{1}^{\prime}=\{z \in D:\|z-q\| \leq r\}, B_{2}^{\prime}=\{z \in$ $D:\|z-q\| \leq 2 r\}, M^{\prime}=\sup _{n}\left\{\left\|u_{n}-q\right\|\right\}$. Next, we prove that $z_{n} \in B_{1}^{\prime}$ for any $n \geq 0$ by induction. First $z_{0} \in B_{1}^{\prime}$ is obvious. Suppose that $z_{n} \in B_{1}^{\prime}$ holds. We prove that $z_{n+1} \in B_{1}^{\prime}$. If it is not the case, then $\left\|z_{n+1}-q\right\|>r$. By uniformly continuity of $J$ on bounded subset, we choose $\epsilon_{0}=\Phi(r / 2) / 16 L(1+2 r)$, there exists $\delta>0$ such that $\|J x-J y\|<\epsilon_{0}$ when $\|x-y\|<$ $\delta$, for all $x, y \in B_{2}^{\prime}$. Now denote

$$
\tau_{0}=\min \left\{\frac{r}{2\left[L(1+r)+2 r+M^{\prime}\right]}, \frac{\delta}{2\left[L(1+r)+2 r+M^{\prime}\right]}, \frac{\Phi(r / 2)}{8 r^{2}}, \frac{\Phi(r / 2)}{24 L(1+2 r)}, \frac{\Phi(r / 2)}{16 M^{\prime} r}\right\} .
$$

Since $a_{n}, c_{n}, k_{n}-1 \rightarrow 0$ as $n \rightarrow \infty$, and $c_{n}=o\left(a_{n}\right)$, without loss of generality, let $0 \leq a_{n}, c_{n}, k_{n}-$ $1 \leq \tau_{0}, c_{n}<a_{n} \tau_{0}$ for any $n \geq 0$. Then we have the following estimates from (1.8):

$$
\begin{aligned}
\left\|z_{n}-T^{n} z_{n}\right\| & \leq\left\|z_{n}-q\right\|+\left\|T^{n} z_{n}-q\right\| \\
& \leq r+L(1+r), \\
\left\|z_{n}-q\right\| & \geq\left\|z_{n+1}-q\right\|-a_{n}\left\|T^{n} z_{n}-z_{n}\right\|-c_{n}\left\|u_{n}-z_{n}\right\| \\
& >r-a_{n}[r+L(1+r)]-c_{n}\left(r+M^{\prime}\right) \\
& \geq r-\tau_{0}\left[L(1+r)+2 r+M^{\prime}\right] \\
& \geq \frac{r}{2}
\end{aligned}
$$




$$
\begin{aligned}
\left\|z_{n+1}-q\right\| & \leq\left(1-a_{n}-c_{n}\right)\left\|z_{n}-q\right\|+a_{n}\left\|T^{n} z_{n}-q\right\|+c_{n}\left\|u_{n}-q\right\| \\
& \leq r+\tau_{0}\left[L(1+r)+M^{\prime}\right] \\
& \leq 2 r, \\
\left\|\left(z_{n+1}-q\right)-\left(z_{n}-q\right)\right\| & \leq a_{n}\left\|T^{n} z_{n}-z_{n}\right\|+c_{n}\left\|u_{n}-z_{n}\right\| \\
& \leq a_{n}[r+L(1+r)]+c_{n}\left(r+M^{\prime}\right) \\
& \leq \tau_{0}\left[L(1+r)+2 r+M^{\prime}\right] \\
& \leq \frac{\delta}{2}<\delta .
\end{aligned}
$$

Therefore, $\left\|J\left(z_{n+1}-q\right)-J\left(z_{n}-q\right)\right\|<\epsilon_{0}$.

Using Lemma 1.6 and formulas above, we obtain

$$
\begin{aligned}
\left\|z_{n+1}-q\right\|^{2} \leq & \left(1-a_{n}\right)^{2}\left\|z_{n}-q\right\|^{2}+2 a_{n}\left\langle T^{n} z_{n}-q, J\left(z_{n+1}-q\right)-J\left(z_{n}-q\right)\right\rangle \\
& +2 a_{n}\left\langle T^{n} z_{n}-q, J\left(z_{n}-q\right)\right\rangle+2 c_{n}\left\langle u_{n}-q, J\left(z_{n+1}-q\right)\right\rangle \\
\leq & \left(1-a_{n}\right)^{2}\left\|z_{n}-q\right\|^{2}+2 a_{n}\left\|T^{n} z_{n}-q\right\| \cdot\left\|J\left(z_{n+1}-q\right)-J\left(z_{n}-q\right)\right\| \\
& +2 a_{n}\left[k_{n}\left\|z_{n}-q\right\|^{2}-\Phi\left(\left\|z_{n}-q\right\|\right)\right]+2 c_{n}\left\|u_{n}-q\right\| \cdot\left\|z_{n+1}-q\right\| \\
\leq & \left(1-a_{n}\right)^{2} r^{2}+4 a_{n} L(1+2 r) \epsilon_{0} \\
& +2 a_{n}\left[k_{n}\left\|z_{n}-q\right\|^{2}-\Phi\left(\left\|z_{n}-q\right\|\right)\right]+4 c_{n} M^{\prime} r \\
\leq & \left(1-a_{n}\right)^{2} r^{2}+4 a_{n} L(1+2 r) \epsilon_{0}+2 a_{n}\left[k_{n} r^{2}-\Phi\left(\frac{r}{2}\right)\right]+4 c_{n} M^{\prime} r \\
= & r^{2}+2 a_{n}\left[\frac{a_{n}}{2} r^{2}+2 L(1+2 r) \epsilon_{0}+\left(k_{n}-1\right) r^{2}+\frac{2 c_{n} M^{\prime} r}{a_{n}}\right]-2 a_{n} \Phi\left(\frac{r}{2}\right) \\
\leq & r^{2}+2 a_{n}\left[\frac{\Phi(r / 2)}{2}-\Phi\left(\frac{r}{2}\right)\right] \\
\leq & r^{2}-a_{n} \Phi\left(\frac{r}{2}\right) \\
\leq & r^{2},
\end{aligned}
$$

this is a contradiction. Thus $z_{n+1} \in B_{1}^{\prime}$, that is, $\left\{z_{n}\right\}$ is a bounded sequence, so $\left\{T^{n} z_{n}\right\}$ is also bounded. Denote $M_{0}=\sup _{n}\left\{\left\|z_{n}-q\right\|\right\}+\sup _{n}\left\{\left\|T^{n} z_{n}-q\right\|\right\}+\sup _{n}\left\{\left\|u_{n}-q\right\|\right\}$.

Step 3 . We prove $\left\|z_{n}-q\right\| \rightarrow 0$ as $n \rightarrow \infty$. 
Again using Lemma 1.6, we have

$$
\begin{aligned}
\left\|z_{n+1}-q\right\|^{2} \leq & \left(1-a_{n}-c_{n}\right)^{2}\left\|z_{n}-q\right\|^{2}+2 a_{n}\left\langle T^{n} z_{n}-q, J\left(z_{n+1}-q\right)\right\rangle \\
& +2 c_{n}\left\langle u_{n}-q, J\left(z_{n+1}-q\right)\right\rangle \\
\leq & \left(1-a_{n}\right)^{2}\left\|z_{n}-q\right\|^{2}+2 a_{n}\left\langle T^{n} z_{n}-q, J\left(z_{n+1}-q\right)-J\left(z_{n}-q\right)\right\rangle \\
& +2 a_{n}\left\langle T^{n} z_{n}-q, J\left(z_{n}-q\right)\right\rangle+2 c_{n}\left\|u_{n}-q\right\| \cdot\left\|z_{n+1}-q\right\| \\
\leq & \left(1-a_{n}\right)^{2}\left\|z_{n}-q\right\|^{2}+2 a_{n} M_{0} D_{n} \\
& +2 a_{n}\left[k_{n}\left\|z_{n}-q\right\|^{2}-\Phi\left(\left\|z_{n}-q\right\|\right)\right]+2 c_{n} M_{0}^{2} \\
\leq & \left\|z_{n}-q\right\|^{2}+2 a_{n}\left[\left(k_{n}-1\right) M_{0}^{2}+\frac{a_{n} M_{0}^{2}}{2}+M_{0} D_{n}+\frac{c_{n} M_{0}^{2}}{a_{n}}-\Phi\left(\left\|z_{n}-q\right\|\right)\right] \\
\leq & \left\|z_{n}-q\right\|^{2}+2 a_{n}\left[E_{n}-\Phi\left(\left\|z_{n}-q\right\|\right)\right],
\end{aligned}
$$

where

$$
D_{n}=\left\|J\left(z_{n+1}-q\right)-J\left(z_{n}-q\right)\right\|, \quad E_{n}=\left(k_{n}-1\right) M_{0}^{2}+\frac{a_{n} M_{0}^{2}}{2}+M_{0} D_{n}+\frac{c_{n} M_{0}^{2}}{a_{n}},
$$

and $D_{n}, E_{n} \rightarrow 0$ as $n \rightarrow \infty$.

Set $\inf _{n \geq 0} \Phi\left(\left\|z_{n}-q\right\|\right) /\left(1+\left\|z_{n+1}-q\right\|^{2}\right)=\lambda$, then $\lambda=0$. If it is not the case, we assume that $\lambda>0$. Let $0<\gamma<\min \{1, \lambda\}$, then $\Phi\left(\left\|z_{n}-q\right\|\right) /\left(1+\left\|z_{n+1}-q\right\|^{2}\right) \geq \gamma$, that is, $\Phi\left(\left\|z_{n}-q\right\|\right) \geq$ $\gamma+\gamma\left\|z_{n+1}-q\right\|^{2} \geq \gamma\left\|z_{n+1}-q\right\|^{2}$. Thus, from (2.14) that

$$
\left\|z_{n+1}-q\right\|^{2} \leq\left\|z_{n}-q\right\|^{2}+2 a_{n}\left(E_{n}-\gamma\left\|z_{n+1}-q\right\|^{2}\right),
$$

which implies that

$$
\begin{aligned}
\left\|z_{n+1}-q\right\|^{2} & \leq \frac{1}{1+2 a_{n} \gamma}\left\|z_{n}-q\right\|^{2}+\frac{2 a_{n} E_{n}}{1+2 a_{n} \gamma} \\
& =\left(1-\frac{2 a_{n} \gamma}{1+2 a_{n} \gamma}\right)\left\|z_{n}-q\right\|^{2}+\frac{2 a_{n} E_{n}}{1+2 a_{n} \gamma} .
\end{aligned}
$$

Let $\rho_{n}=\left\|z_{n}-q\right\|^{2}, \lambda_{n}=2 a_{n} \gamma /\left(1+2 a_{n} \gamma\right), \sigma_{n}=2 a_{n} E_{n} /\left(1+2 a_{n} \gamma\right)$. Then we get that

$$
\rho_{n+1} \leq\left(1-\lambda_{n}\right) \rho_{n}+\sigma_{n}
$$

Applying Lemma 1.7, we get that $\rho_{n} \rightarrow 0$ as $n \rightarrow \infty$. This is a contradiction and so $\lambda=0$. Therefore, there exists an infinite subsequence such that $\Phi\left(\left\|z_{n_{i}}-q\right\|\right) /\left(1+\left\|z_{n_{i}+1}-q\right\|^{2}\right) \rightarrow 0$ as $i \rightarrow \infty$. Since $0 \leq \Phi\left(\left\|z_{n_{i}}-q\right\|\right) /\left(1+M_{0}^{2}\right) \leq \Phi\left(\left\|z_{n_{i}}-q\right\|\right) /\left(1+\left\|z_{n_{i}+1}-q\right\|^{2}\right)$, then $\Phi\left(\left\|z_{n_{i}}-q\right\|\right) \rightarrow 0$ 
as $i \rightarrow \infty$. In view of the strictly increasing and continuity of $\Phi$, we have $\left\|z_{n_{i}}-q\right\| \rightarrow 0$ as $i \rightarrow \infty$. Let $\varepsilon \in(0,1)$ be any given, there exists $n_{i_{0}}$ such that $\left\|z_{n_{i}}-q\right\|<\epsilon, a_{n_{i}}, a_{n}<$ $\min \left\{\epsilon / 4 L\left(1+M_{0}\right), \epsilon / 8 M_{0}\right\}, c_{n_{i}}, c_{n}<\epsilon / 16 M_{0}, E_{n_{i}}, E_{n}<\Phi(\epsilon / 2) / 2$, for any $n_{i}, n \geq n_{i_{0}}$. First, we want to prove $\left\|z_{n_{i}+1}-q\right\|<\epsilon$. Suppose it is not this case, then $\left\|z_{n_{i}+1}-q\right\| \geq \epsilon$. Using (1.8), we may get the following estimates:

$$
\begin{aligned}
\left\|z_{n_{i}}-q\right\| & \geq\left\|z_{n_{i}+1}-q\right\|-a_{n_{i}}\left\|T^{n} z_{n_{i}}-q\right\|-a_{n_{i}}\left\|z_{n_{i}}-q\right\|-c_{n_{i}}\left\|u_{n_{i}}-q\right\| \\
& \geq \epsilon-a_{n_{i}} L\left(1+M_{0}\right)-\left(a_{n_{i}}+2 c_{n_{i}}\right) M_{0} \\
& >\frac{\epsilon}{2} .
\end{aligned}
$$

Since $\Phi$ is strictly increasing, then (2.32) leads to $\Phi\left(\left\|z_{n_{i}}-q\right\|\right) \geq \Phi(\epsilon / 2)$. From (2.27), we have

$$
\begin{aligned}
\left\|z_{n_{i}+1}-q\right\|^{2} & \leq\left\|z_{n_{i}}-q\right\|^{2}+2 a_{n_{i}}\left[E_{n_{i}}-\Phi\left(\left\|z_{n_{i}}-q\right\|\right)\right] \\
& <\epsilon^{2}+2 a_{n_{i}}\left[\frac{1}{2} \Phi\left(\frac{\epsilon}{2}\right)-\Phi\left(\frac{\epsilon}{2}\right)\right] \\
& \leq \epsilon^{2}-\Phi\left(\frac{\epsilon}{2}\right) a_{n_{i}} \\
& \leq \epsilon^{2}
\end{aligned}
$$

is a contradiction. Hence, $\left\|z_{n_{i}+1}-q\right\|<\epsilon$. Suppose that $\left\|z_{n_{i}+m}-q\right\|<\epsilon$ holds. Repeating the above course, we can easily prove that $\left\|z_{n_{i}+m+1}-q\right\|<\epsilon$ holds. Therefore, for any $m$ and $n_{i} \geq n_{0}$, we obtain that $\left\|z_{n_{i}+m}-q\right\|<\epsilon$, which means $\left\|z_{n}-q\right\| \rightarrow 0$ as $n \rightarrow \infty$. This completes the proof.

Theorem 2.4. Let $E$ be an arbitrary uniformly smooth real Banach space, let $D$ be a nonempty closed convex subset of $E$, and let $T: D \rightarrow D$ be a uniformly generalized Lipschitz generalized asymptotically $\Phi$-strongly pseudocontractive mapping with $q \in F(T) \neq \emptyset$. Let $\left\{a_{n}\right\},\left\{b_{n}\right\},\left\{c_{n}\right\},\left\{d_{n}\right\}$ be four real sequences in $[0,1]$ and satisfy the conditions (i) $a_{n}+c_{n} \leq 1, b_{n}+d_{n} \leq 1$; (ii) $a_{n}, b_{n}, d_{n} \rightarrow 0$ as $n \rightarrow \infty$ and $c_{n}=o\left(a_{n}\right)$; (iii) $\sum_{n=0}^{\infty} a_{n}=\infty$. For some $x_{0} \in D$, let $\left\{v_{n}\right\},\left\{w_{n}\right\}$ be two arbitrary bounded sequences in $D$, and let $\left\{x_{n}\right\}$ be Ishikawa iterative sequence with errors defined by (1.7). Then (1.7) converges strongly to the unique fixed point $q$ of $T$.

Proof. By Theorems 2.3 and 2.2, we obtain directly the result of Theorem 2.4.

Remark 2.5. Our Theorem 2.2 extends and improves Theorem 3.1 of [4] from the bounded range of $T$ to uniformly generalized Lipschitz mapping, and the proof course of Theorem 2.2 is quite different from that of [4].

\section{References}

[1] K. Goebel and W. A. Kirk, "A fixed point theorem for asymptotically nonexpansive mappings," Proceedings of the American Mathematical Society, vol. 35, pp. 171-174, 1972.

[2] J. Schu, "Iterative construction of fixed points of asymptotically nonexpansive mappings," Journal of Mathematical Analysis and Applications, vol. 158, no. 2, pp. 407-413, 1991. 
[3] B. E. Rhoades, "Comments on two fixed point iteration methods," Journal of Mathematical Analysis and Applications, vol. 56, no. 3, pp. 741-750, 1976.

[4] V. Colao, "On the convergence of iterative processes for generalized strongly asymptotically $\varphi$ pseudocontractive mappings in Banach spaces," Journal of Applied Mathematics, vol. 2012, Article ID 563438, 18 pages, 2012.

[5] S. S. Chang, "Some results for asymptotically pseudo-contractive mappings and asymptotically nonexpansive mappings," Proceedings of the American Mathematical Society, vol. 129, no. 3, pp. 845-853, 2001.

[6] C. E. Chidume and C. O. Chidume, "Convergence theorem for zeros of generalized Lipschitz generalized phi-quasi-accretive operators," Proceedings of the American Mathematical Society, vol. 134, no. 1, pp. 243-251, 2006.

[7] K. Deimling, Nonlinear Functional Analysis, Springer, New York, NY, USA, 1980.

[8] X. Weng, "Fixed point iteration for local strictly pseudo-contractive mapping," Proceedings of the American Mathematical Society, vol. 113, no. 3, pp. 727-731, 1991. 


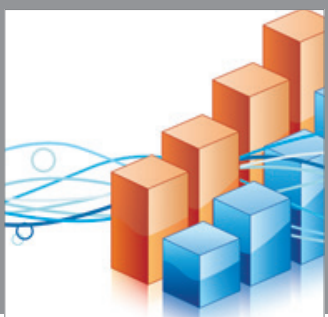

Advances in

Operations Research

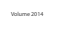

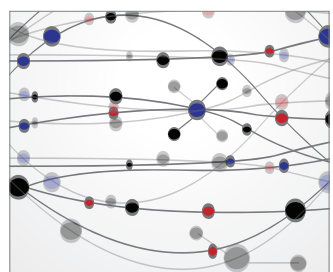

\section{The Scientific} World Journal
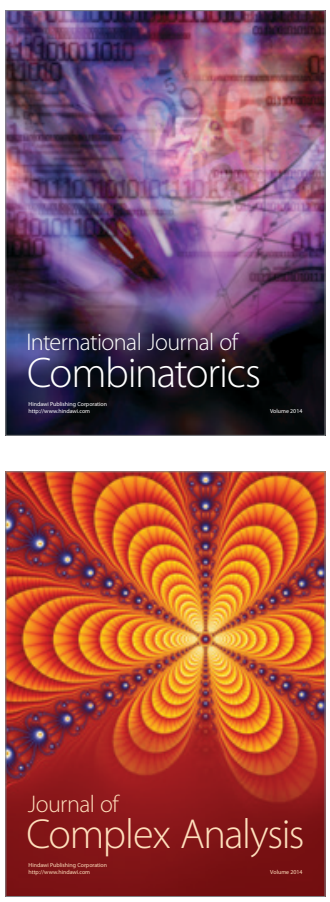

International Journal of

Mathematics and

Mathematical

Sciences
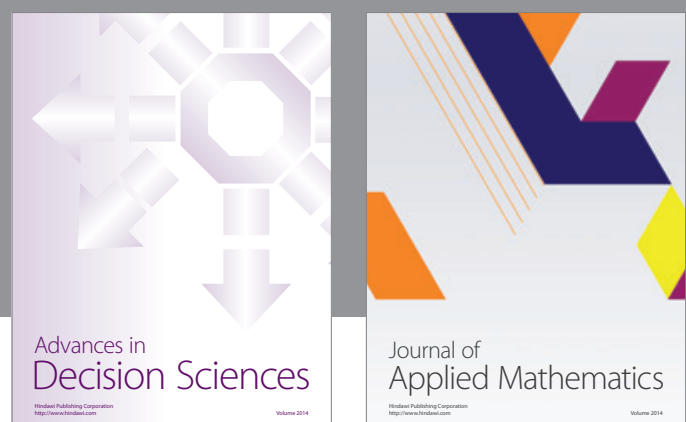

Journal of

Applied Mathematics
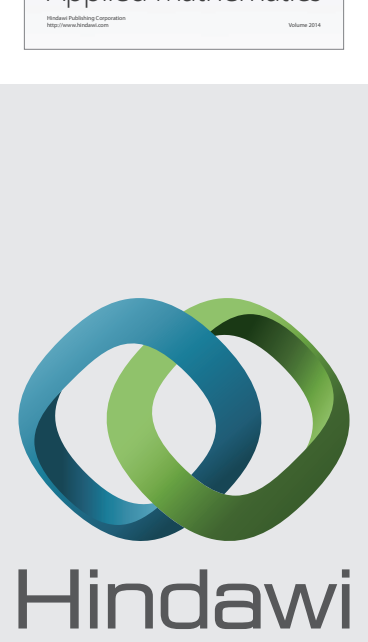

Submit your manuscripts at http://www.hindawi.com
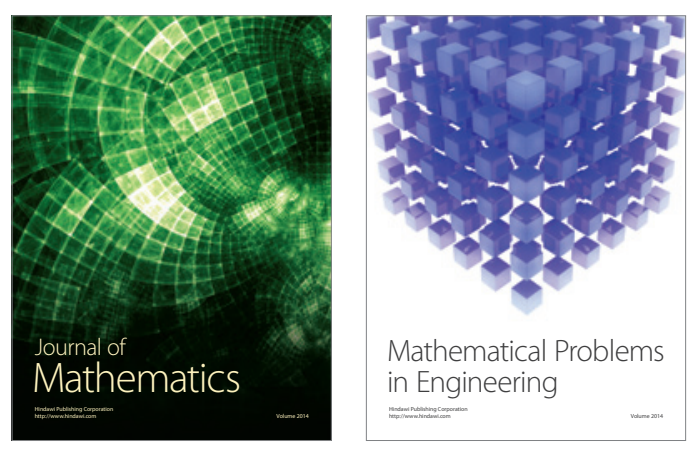

Mathematical Problems in Engineering
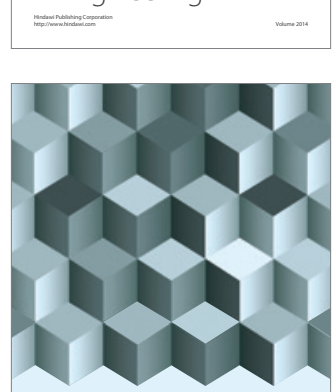

Journal of

Function Spaces
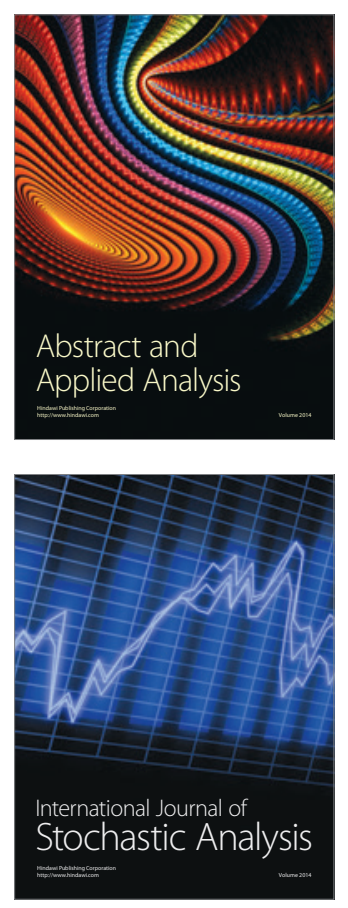

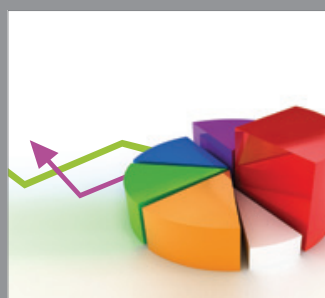

ournal of

Probability and Statistics

Promensencen
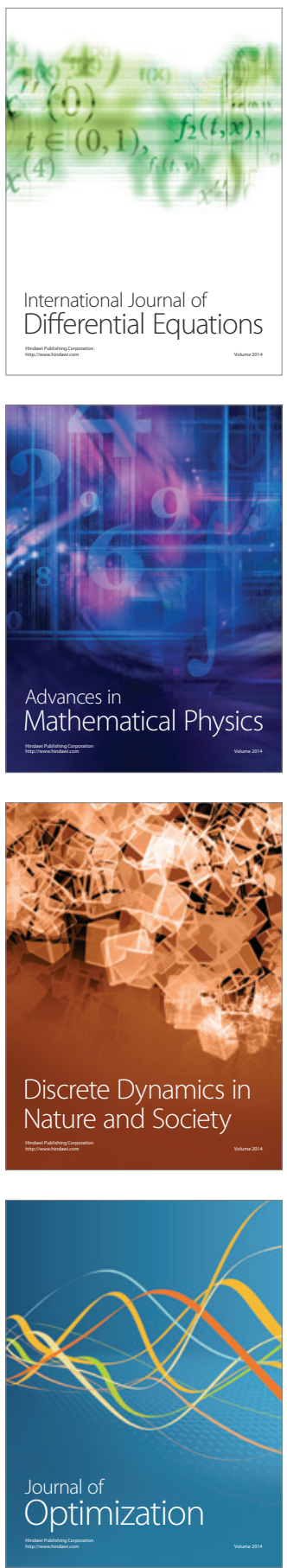